\title{
EMBEDDINGS BETWEEN WEIGHTED CESÀRO FUNCTION SPACES
}

\author{
TUĞÇE ÜNVER
}

Abstract. In this paper, we give the characterization of the embeddings between weighted Cesàro function spaces. The proof is based on the duality technique, which reduces this problem to the characterizations of some direct and reverse Hardy-type inequalities and iterated Hardy-type inequalities.

Mathematics subject classification (2010): 46E30, 26D10, 47G10, 47B38.

Keywords and phrases: Cesàro and Copson function spaces, embeddings, weighted inequalities, Hardy and Copson operators, iterated operators.

\section{REFERENCES}

[1] S.V. AstashKin On the geometric properties of Cesàro spaces. Russian, with Russian summary, Mat. Sb. 2034 (2012), 61-80.

[2] S.V. Astashin and L. Maligranda, Cesàro function spaces fail the fixed point property, Proc. Amer. Math. Soc. 13612 (2008), 4289-4294.

[3] S.V. Astashkin And L. Maligranda, Structure of Cesàro function spaces, Indag. Math. (N.S.). 203 (2009), 329-379.

[4] S.V. Astashin And L. Maligranda, Rademacher functions in Cesàro type spaces, Studia Math. 1983 (2010), 235-247.

[5] S.V. Astashin And L. Maligranda, Geometry of Cesàro function spaces, Russian. Funktsional. Anal. i Prilozhen. 451 (2011) 79-82, translation in Funct. Anal. Appl. 451 (2011), 64-68.

[6] S.V. AstaShKin AND L. MALigRAndA, Interpolation of Cesàro sequence and function spaces, Studia Math. 2151 (2013), 39-69.

[7] S.V. Astashin AND L. Maligranda, A short proof of some recent results related to Cesàro function spaces, Indag. Math. (N.S.) 243 (2013), 589-592.

[8] S.V. AstashKin AND L. MALigRAnda, Interpolation of Cesàro and Copson spaces, Banach and function spaces IV (ISBFS 2012). Yokohama Publ., Yokohama. 123-133, 2014.

[9] S.V. Astashrin AND L. MaligRanda, Structure of Cesàro function spaces: a survey, Banach Center Publ. 102 (2014), 13-40.

[10] S. Barza, A.N. Marcoci And L.G. Marcoci, Factorizations of weighted Hardy inequalities, Bull. Braz. Math. Soc. (N.S.) 494 (2018), 915-932.

[11] G. Bennett, Factorizing the classical inequalities, Mem. Amer. Math. Soc. 120, 1996.

[12] W.D. Evans, A. Gogatishvili And B. OpIC, The reverse Hardy inequality with measures, Math. Inequal. Appl. 111 (2008), 43-74.

[13] A. Gogatishvili, R.Ch. Mustafayev and L.-E. Persson, Some new iterated Hardy-type inequalities, J. Funct. Spaces Appl. (2012).

[14] A. Gogatishvili and R.CH. Mustafayev, Weighted iterated Hardy-type inequalities, Math. Inequal. Appl. 203 (2017), 683-728.

[15] A. Gogatishvili And R.CH. Mustafayev, Iterated Hardy-type inequalities involving suprema, Math. Inequal. Appl. 204 (2017), 901-927.

[16] A. Gogatishvili, R.Ch. Mustafayev and T. ÜnVer, Embeddings between weighted Copson and Cesàro function spaces, Czechoslovak Math. J. 67 (142) 4 (2017), 1105-1132.

[17] A. Gogatishvili, R.Ch. Mustafayev and T. ÜnVer, Pointwise Multipliers between weighted Copson and Cesàro function spaces, Math. Slovaca 696 (2019), 1303-1328. 
[18] A. GogatishVili And L. Pick, Embeddings and duality theorems for weak classical Lorentz spaces, Canad. Math. Bull. 491 (2006), 82-95.

[19] A. Gogatishvili, B. OPIC AND L. PICK, Weighted inequalities for Hardy-type operators involving suprema, Collect. Math. 573 (2006), 227-255.

[20] K.-G. Grosse-ERdMANn, The blocking technique, weighted mean operators and Hardy's inequality, Lecture Notes in Mathematics. $1679 \mathrm{x}+114$, Springer-Verlag, Berlin, 1998.

[21] B.D. Hass ARD And D. A. Hussein, On Cesàro function spaces, Tamkang J. Math. 4 (1973), 19-25.

[22] A. KAMIŃSKA AND D. KubiaK, On the dual of Cesàro function space, Nonlinear Anal. 755 (2012), 2760-2773.

[23] M. KŘEPELA, Integral conditions for Hardy-type operators involving suprema, Collect. Math. 681 (2017), 21-50.

[24] M. Ǩ̌EPEla AND L. PICK, Weighted inequalities for iterated Copson integral operators, Studia Math. 2531 (2020), 163-197.

[25] K. LeŚniK AND L. Maligranda, Symmetrization, factorization and arithmetic of quasi-Banach function spaces, J. Math. Anal. Appl. 4702 (2019), 1136-1166.

[26] R. Mustafayev, On weighted iterated Hardy-type inequalities, Positivity. 221 (2018), 275-299.

[27] B. Opic And A. Kufner, Hardy-type inequalities, Pitman Research Notes in Mathematics Series. 219 xii+333, Longman Scientific \& Technical, Harlow, 1990.

[28] J.-S. SHIUE, A note on Cesàro function space, Tamkang J. Math. 12 (1970), 91-95.

[29] P.W. SY, W.Y. Zhang AND P.Y. LeE, The dual of Cesàro function spaces, Glas.Mat. Ser. III. 22 (42) 1 (1987), 103-112. 